M as pero, G., Notes sur quelques points de Grammaire et d'Histoire 8. Z. f. ä. Spr. ù. A., S. $116 \mathrm{ff}$.

+ Noordtzy, M., Egyptologie en Assyriologie in betrekking tot de geloofwaardigheid des ouden testaments. Utrecht $1881.48 \mathrm{~S} .8^{\circ}$

$\dagger$ Oliphant, L., the Land of Khemi : Up and Down the Middle Nile. With Illustr. London 1882. 266 S. $8^{\circ}$.

† Piehl, Karl, Petites études égyptologiques. Stockholm 1881. 63 S. $8^{\circ}$.

Derselbe, Inscription de la XII dynastie s. Z. f. a. Spr. u. A. 1881 , S. $19 \mathrm{ff}$.

+ Pierret, P., Le decrét trilingue de Canope. Paris 1882. XVI. 49 S. $4^{0}$.

+ Poit ou, E., Un hiver en Égypte. Tours 1881. 397 S. $4^{0}$.

+ Poole, S. L, Egypt. With Illustrations. London 1881. 204 S. $8^{0}$.

$+\mathrm{R}$ aw lins on, G., History of Ancient Egypt. London 1881. 2 vols. 830 S. $8^{\circ}$.

$\dagger$ Records of the Past. Vol. 12. Egyptian Texts. London 1881. 176 S. $8^{\circ}$.

Rodet, L., les prétendus problèmes d'algèbre du manuel du calculateur Egyptien s. Journal Asiatique 1881, S. $184 \mathrm{ff} ., 390 \mathrm{ff}$.

+ Sarrasi, l'antique Orient dévoilé par les hiéroglyphes et les inscriptions cunéiformes. Toulouse 1881 . $264 \mathrm{~S} .8^{\circ}$.

+ Wils on, E., The Egypt of the Past. With 46 Illustr. London 1881. 488 s. $8^{\circ}$.

Bernus, A., Notice bibliographique sur Richard Simon. (Extrait de l'éssai de Bibliographie Oratorienne par A. M. P. In gold.) Bâle 1882. 48 s. $8^{\circ}$.

\title{
Zur Nachricht.
}

Als Bogen 4 dieses Heftes gedruckt vorlag, erschien A. Dillmann's ${ }_{n}$ Die Genesis f. d. 4. Aufl. nach A. Knobel erklärt". Da der Verf. darin seine Ansichten über Gen. 48 und die damit zusammenhängenden Abschnitte in mehreren nicht unwesentlichen Punkten geändert hat, insbesondere über 48,8 ff. in der Hauptsache dieselbe Anschauung vertritt, die auf S. 57-61, Abs. 1 dieses Heftes entwickelt wird, so ersuchte mich gleich damals Prof. Budde, ihm entweder Nachträge zu gestatten oder eine diesbezügliche Erklärung aufzunehmen. Das letztere schien mir richtiger, und so erkläre ich demzufolge : dafs der Aufsatz von Prof. Budde am 10. Oct. 1882 zur Post gegeben worden ist, demnach nur die 3. Aufl. des Knobel-Dillmann'schen Commentars darin berücksichtigt werden konnte, dafs der Verf. auch während des Druckes nichts mit Bezug auf die 4. Auflage geändert oder hinzugesetzt hat. Die von Prof. Budde S. 56 erwähnte Correspondenz mit mir fiel in die erste Hälfte des Monats August.

Giefsen, den 28. December 1882.

B. Stade. 


\section{Neuer Versuch einer Chronologie der hebräischen Könige. \\ Von Adolf Kamphausen.}

1. Ehe ich meinen Versuch, die Regierungsjahre der einzelnen hebräischen Könige zu fixiren, dem Leser in einer kurzen Tabelle vorlege, welche ich später an einem andern Orte zu begründen hoffe, möchte ich zur Vergleichung die interessanten Aufstellungen von $\mathrm{Max} \mathrm{Duncker}$ und $\mathrm{Ju}$ lius Wellhausen vorausschicken. Nach dem zweiten Bande von Duncker's in vieler Hinsicht ausgezeichneten Geschichte des Alterthums (5. Aufl. Leipzig 1878), worin besonders S. 89.182 f. 270 ff. 375 zu vergleichen sind, ergeben sich folgende Regierungsjahrzahlen :

Saul 1055-1033 v. Chr.

David 1033-993

Salomo 993-953

$\begin{array}{lclc}\text { * Rehabeam } & 953-932 & \text { * Jerobeam I. } & 953-927 \\ \text { Abiam } & 932-929 & & \\ \text { *Asa } & 929-873 & \text { Nadab } & 927-925 \\ & & \text { Baesa } & 925-901 \\ & & \text { Ela } & 901-899 \\ & & \text { Simri } & 899 \\ & & \text { * Omri } & 899-875 \\ \text { Josaphat } & 873-848 & \text { Ahab } & 875-853 \\ \text { " Jehoram } & 848-844 & \text { Ahasja } & 853-851 \\ \text { Ahasjahu } & 844-843 & \text { * Joram } & 851-843 \\ \text { Athalja } & 843-837 & \text { Jehu } & 843-815 \\ \text { Jehoas } & 837-797 & \text { Joahas } & 815-798 \\ \text { *Amazja } & 797-792 & \text { * Joas } & 798-790\end{array}$

Zeitschrift f. d. alttest. Wiss. Jahrgang 3. 1883. 
Usia

* Jotham

* Ahas

* Hiskia

Manasse

Amon

Josia

Jehoahas

Jojakim

Jechonja

Zedekia
$792-740$

$740-734$

$734-728$

$728-697$

697-642

642-640

$640-609$

609

608-597

597

Duncker, der S. 182 wohl nur durch ein Druckversehen Asa, den Sohn (1 Kön. 15, 8) des Abiam, zu dessen Bruder macht, nennt selbst den judäischen König schon Jehoram, wie auch andere Gelehrte thun; ich habe zur weiteren Unterscheidung der in beiden Reichen gleichnamigen Könige die Namen Ahasja, Joahas und Joas für Israel beibehalten, dagegen für Juda die Formen Ahasjahu, Jehoahas und Jehoas oben zur Anwendung gebracht. Während J. Oppert (Gött. gel. Anz. 1881, Stück 4) in seiner Anzeige der Schriften von V. Floigl und F. Hommel sich rühmte, dafs er in den 171 chronologischen Aussagen des biblischen Textes nur 8 Schreibfehler gefunden habe, dünkt Duncker mit vollem Rechte dieser Ruhm nicht fein. Ich kann es nur billigen, dafs D uncker die hebräischen Königszahlen mit Hülfe der assyrischen Inschriften verbessern will, und ich theile seine Zuversicht, dafs in den biblischen Zahlen genug glaubwürdiges Material erhalten ist, um den Versuch einer solchen Correctur mit Aussicht auf Erfolg überhaupt möglich zu machen. Es fehlt sogar nicht an guten geschichtlichen Gründen dafür, dafs wir die Regierungszeit des Saul, David und Salomo annähernd zu 
einem Jahrhundert berechnen. Noch berechtigter aber erscheint mir die Ansicht, dafs wir für die Könige der getheilten Reiche ziemlich genaue Regierungszahlen auf Grund der Zuverlässigkeit gewisser biblischer Angaben berechnen können. D un cker kann nicht meinen, dafs alle biblischen Zahlen gleichwerthig seien, denn S. 183 Anm. erklärt er es für eine aussichtslose Aufgabe, die Divergenzen der Vergleichungen der beiden Königsreihen ausgleichen zu wollen, und doch hält er sich berechtigt, die biblischen Angaben für die Regierungsdauer der einzelnen Könige, allerdings mit der Ausnahme von 13 Zahlen, seiner auf historische Wahrscheinlichkeit Anspruch machenden Aufstellung zu Grunde zu legen. Von diesen 13 Ausnahmefällen kommen 7 auf die judäischen Könige, denn Duncker giebt dem Rehabeam 21 Regierungsjahre statt der biblischen 17, dem Asa 56 statt 41, dem Jehoram 4 statt 8, dem Amazja 5 statt 29, dem Jotham 6 statt 16, dem Ahas 6 statt 16, dem Hiskia 31 statt 29. Die übrigen 6 der in der Tabelle oben durch ein Sternchen angedeuteten Aenderungen vertheilen sich auf die Könige Israels so, dafs D uncker dem Jerobeam I. 26 Regierungsjahre giebt statt der biblischen 22, dem Omri 24 statt 12, dem Joram 8 statt 12, dem Joas 8 statt 16, dem Pekah 2 oder 3 statt 20, endlich dem Hosea 12 statt 9 . Das Bedenkliche dieser 13 Aenderungen verhehlt sich Duncker selbst nicht; aber er sagt S. 272 Anm. : ${ }_{\text {So }}$ gewaltsam diese Annahmen den überlieferten Zahlen der Bücher der Könige gegenüber erscheinen, sie geben sich nur für erzwungene Hypothesen und lassen wenigstens die überlieferten Thatsachen stehen, während die Uebereinstimmung, welche durch Unterstellung von Mitregentschaften, einer ersten und zweiten Regierung des zweiten Jerobeam, eines ersten und zweiten Menahem, einer ersten und zweiten Regierung Pekahs für die Listen von Juda und Israel herbeigeführt werden soll, die Ueberlieferung selbst alterirt und die Concordanz mit der Epo- 
nymenliste dennoch nicht zu Stande bringt.“ Gewifs, hätte ich nur die Wahl zwischen Duncker und Oppert, so würde ich mich für den ersteren Gelehrten entscheiden müssen; finde ich doch bei Duncker trotz einer gewissen Unterschätzung biblischer Zahlen, welche er sich meines Erachtens zu Schulden kommen lärst, dennoch, um mit meinem römisch-katholischen Collegen Fr. Ka ulen (Assyrien und Babylonien nach den neuesten Entdeckungen. Freiburg im Breisgau 1882, S. 203) zu sprechen, „den Geist, der die deutsche Wissenschaft beseelt, während nur 0 ppert den Muth hat, die Angaben der Bibel für unantastbar zu erklären."

Auf eine erschöpfende Kritik von Duncker's Tabelle will ich hier nicht eingehen, sondern den Leser vorläufig auf eine Anzeige verweisen, welche ich von Eb. Schrader's wichtigem Buche „Die Keilinschriften und das Alte Testament. 2. Aufl. Giefsen, $1883^{\text {« }}$ für die Historische Zeitschrift von H. v. Sybel geschrieben habe. Ich zähle hier nur einige Punkte auf, in welchen ich glaube, von Duncker abweichen zu müssen : 1) Das Jahr 842, in welchem Jehn dem Salmanasser II. Tribut zahlte, halte ich für Jehu's erstes Regierungsjahr und finde in dieser Tributzahlung keinen Beweis für Duncker's Meinung, dafs kein späteres Jahr als 843 das erste Jahr Jehu's sein könne. 2) In der ungefähren Uebereinstimmung der 95 judäischen und der 98 israelitischen Jahre, welche die Bibel von der Reichsspaltung bis zum Sturze des Hauses Omri angiebt, erblicke ich eine werthvolle Beglaubigung der für diese Periode gegebenen einzelnen Regierungszahlen, halte aber die Zahl 95 für die genauere. Es ist eine historisch nahe liegende Annahme, dafs die je 2 Jahre, welche dem Nadab, dem Trunkenbold Ela und dem Pekahja gegeben sind, nicht als volle Jahre gerechnet werden dürfen, da diese Söhne der gewifs nicht unfähigen Usurpatoren Jerobeam I., Baesa und Menahem wahrscheinlich als unbedeutende 
Menschen bald aus dem Wege geräumt wurden. Uebrigens verdient die Zahl 95 schon darum, weil sie die judäische und dennoch die kleinere ist, den Vorzug. 3) Während ich durch Hinzurechnung der 95 zu 842 für den gleichzeitigen Regierungsanfang des Jerobeam I. und Rehabeam 937 gewinne, zählt D uncker zu 843 nicht nur 98 hinzu, sondern vergröfsert diese 941 noch um weitere 12 Jahre, die er der nach der Bibel 12 jährigen Regierung des Omri um der Bedeutung willen, welche diese Regierung bei den Moabitern und Assyrern habe, zulegen zu müssen glaubt. Omri und sein Sohn Ahab konnten sich in den 34 biblischen. Jahren Ruhm genug bei den Nachbarvölkern erwerben. Die 40 Jahre des Mesasteines erledigen sich nicht durch Vergleichung der ständigen $40 \mathrm{Jahre} \mathrm{im} \mathrm{Buche} \mathrm{der}$ Richter, sondern durch die Annahme, dafs Omri schon als Feldhauptmann des Baesa den Bezirk von Medeba für Israel gewonnen hat. Diese Hypothese scheint mir werthvoller als die Annahme eines gegen 5 Jahre dauernden Bürgerkriegs zwischen Omri und Thibni (vgl. Riehm in seinem Handwörterbuch s. v. Omri). Dieser „längere Bürgerkrieg“ gehört in dieselbe Klasse mit den angeblichen Interregnen und dem Zusammenregieren von Josaphat und Jehoram, d. h. er beruht auf den historisch werthlosen, nur durch Rechnung gewonnenen Synchronismen der Regierungsantritte. 4) Nach dem historisch höchst werthvollen allgemeinen Synchronismus des vorexilischen (vgl. Bleek-Wellhausen § 133) Königsbuches mufs Usia später als Jerobeam II. auf den Thron gekommen sein, ebenso Jotham später als Pekah, während Duncker das Gegentheil annimmt. Der vorexilische Hauptverfasser des Konigsbuches hätte es gewifs nicht unternommen, die Könige beider Reiche in bunter Reihe zu behandeln, hätte er nicht die Absicht gehabt, die streng chronologische Folge einzuhalten, und hätte er nicht in dem ihm zu Gebote stehenden historischen Material auch die nöthigen 
Mittel besessen, um die chronologische Folge wirklich geben zu können. 5) Ich gebe W ellhausen darin Recht, dals der Schreiber der assyrischen Inschrift den Sohn Ahabs Joram mit Ahab selber, der zur Zeit der Schlacht bei Karkar nicht mehr lebte, verwechselt haben mufs, sowie dafs die Annahme von 714 als erstem Regierungsjahre des Hiskia den Vorzug verdient vor der auch von Duncker festgehaltenen Ansicht, wonach 728 das erste Jahr des Hiskia wäre.

2. Gehen wir nun zu Wellhausen's wichtigem Aufsatze über, der mit der Aufschrift „Die Zeitrechnung des Buchs der Könige seit der Theilung des Reiches" 1875 im 20. Bande der Jahrbücher für Deutsche Theologie (S. 607 bis 640 ) erschienen ist. Ich theile daraus $\S 21$ auf S. 638 wörtlich mit : „Zum Schlusse stelle ich die Ergebnisse dieser hypothetischen Correctur der biblischen Zeitrechnung nach den assyrischen Synchronismen in folgender Uebersicht zusammen :
1. Jehu . . . 84 ?
1. Athalia.
7. - . . . 83 ?
1. Joas.
1. Joahaz . . . . 81 ?
23. -
1. Joas . . . . 801
40. -
2. - . . . 800
1. Amasias.
10. - . . . . 792
11. - . . . . 791
9 .
1. Jerobeam . . 785
1. Azarias.
36. - . . . 750
$7 . \quad-$
1. Menahem . . 744
5. - . . . 740
42. -
(1. Jotham).
10. - Antritt Pekah's 735
48. - (7. - )
52. - (11. - )
1. Pekah . . . 734
16. Jotham's. AntrittAhaz'.
2. - Antritt Hosea's 733
1. Ahaz.
9. Hosea . . . . 724
2. -
Fall Samariens . 722
11. Ahaz.
13. Ahaz.

Für die Zeit, in der nur noch Juda allein übrig war, 
würden sich die Ansätze so stellen : $715=20$. Ahaz; $714=1$. Ezechias $; 701=14$. Ezechias $; 686=29$. Ezechias $; 685=1$. Manasse $; 641=45$. Manasse $; 640=1$. Amon; $638=1$. Josias."

Indem ich auch hier auf eine eingehende Kritik in meiner jetzigen kurzen Behandlung der Sache verzichte, beschränke ich mich auf wenige Bemerkungen. Mufs ich auch, was den allgemeinen Synchronismus betrifft, wiederholen, dafs nach der meines Erachtens unanfechtbaren Darstellung des vorexilischen Epitomators Jerobeam II. vor Usia, sowie Pekah vor Jotham den Thron bestiegen hat, so hebe ich es dagegen als einen grofsen wissenschaftlichen Fortschritt hervor, dafs W ellhausen schärfer, als es jemals früher geschehen ist, auf den grofsen Unterschied hingewiesen hat zwischen den vorexilischen Zahlen, die als überlieferte für den Historiker allein in Betracht kommen und die, wenigstens ihrer grofsen Mehrzahl nach, die Vermuthung historischer Treue für sich haben, und zwischen den erst in späteren Zeiten durch gelehrte Rechnung gewonnenen Synchronismen der Regierungsantritte. Werthvoll ist auch der vollkommen gelungene Nachweis, dafs mehrere Bearbeiter des vorexilischen Königsbuches auf Grund der Ueberlieferungszahlen ihre sich oft sehr deutlich widersprechenden Rechnungszahlen zu Stande gebracht haben. Müssen wir nun auch die Rechnungszahlen, obgleich sie nicht alle falsch sind, sämmtlich als für die Chronologie unbrauchbar fallen lassen, so bringt doch diese scheinbare Einbufse den grofsen Gewinn, dals durch dieselbe die Ueberlieferungszahlen um so mehr in unserer Achtung steigen und als wirklich historisches Material zu gelten haben. Mit Recht betont W ellhausen S. 630 „die auffallende gegenseitige Bestätigung" der biblischen und assyrischen Angaben, welche darin liegt, dafs die Bibel den Nimsiden 102 Regierungsjahre gibt, während nach den assyrischen Inschriften in 842 und 738 die Tributzahlungen 
des Jehu und Menahem fallen. Wir dürfen also an den biblischen Ueberlieferungszahlen nicht darum verzweifeln, weil auch in sie während der nachexilischen Zeit künstliche Rechnung eingedrungen ist; vielmehr müssen wir von der Voraussetzung ausgehen, dafs das nachexilische System, welches vom Beginne des salomonischen Tempels bis zur Wiederaufrichtung der Theokratie grade 480 Jahre herausrechnete und zum Theil erst von hier aus die Periode vom Auszuge aus Egypten bis zum Tempelbau zu 480 Jahren bestimmte, von den überlieferten Königszahlen nur wenige verändert haben wird. Ich nehme bei einigen Zahlen unabsichtliche Veränderung an, schliefse aber auch absichtliche Aenderung nicht ganz aus, sondern behaupte nur, dafs sie bona fide geschehen sei. Vielleicht hat $W$ ellhausen die willkürliche Ausmerzung des Pekahja wieder aufgegeben; ich finde auch keinen Grund, dals die Entthronung des Hosea nicht mit der Eroberung von Samaria in dasselbe Jahr fallen sollte.

3. Ich meinerseits bin zu folgender Aufstellung gelangt :

Saul regierte 1037-1018 v. Chr.

David in Juda 1017-1011, ebenso lange Isboseth in Israel;

Davids Herrschaft über Gesammtisrael 1010-978;

Salomo regierte $977-938$.

$\begin{array}{lclc}\text { Rehabeam } & 937-921 & \text { Jerobeam I. } & 937-916 \\ \text { Abiam } & 920-918 & \text { Nadab } & 915-914 \\ \text { Asa } & 917-877 & \text { Baesa } & 914-891 \\ & & \text { Ela } & 891-890 \\ & & \text { Simri } & 890 \\ \text { Josaphat } & 876-852 & \text { Omri } & 890-879 \\ \text { Jehoram } & 851-844 & \text { Ahab } & 878-857 \\ \text { Ahasjahu } & 843 & \text { Joram } & 856-855 \\ \text { Athalja } & 842-837 & \text { Jehu } & 854-843 \\ \text { Jehoas } & 836-797 & \text { Joahas } & 842-815 \\ \text { * Amazja } & 796-778 & \text { Joas } & 797-788 \\ & & & \end{array}$


* Asarja oder Usia 777- 736

Jerobeam II. 781-741 Jotham als Regent 750 - 736 Sacharja und Sallum 741

Jotham als König 735

* Ahas

Hiskia

$734-715$

* Menahem

$740-738$

Pekahja

737-736

* Manasse

$714-686$

* Pekah

$736-730$

Amon

$685-641$

Hosea ,

$730-722$

Josia

Jehoahas

$640-639$

$638-608$

Jojakim

Jechonja

608

Zedekia

607-597

597

$596-586$

Indem ich diese Tabelle der wohlwollenden Prüfung der Leser empfehle, schliefse ich diese kurzen Mittheilungen mit wenigen Bemerkungen. Anstatt der 13 Aenderungen Duncker's halte ich 6 für ausreichend, da ich dem Amazja 19 Jahre zuschreibe statt der biblischen 29, dem Usia 42 statt 52, dem Ahas 20 statt 16, dem Manasse 45 statt 55 , endlich dem Menahem 3 statt 10 und dem Pekah 6 statt 20. Ohne alle Hypothesen kommen wir hier so wenig aus, als auf manchem andern Gebiete der alten Geschichte, und es handelt sich nur um historische Wahrscheinlichkeit, natürlich nicht um die Sicherheit mathematischer Demonstration. Aus voller Ueberzeugung aber theile ich die Ansicht Duncker's, dafs wir uns hier auf wesentlich historischem Boden befinden, so dafs ich die Ueberstürzungen der neuesten alttestamentlichen Kritik lebhafter beklagen mufs, als die mehr unschädlichen Liebhabereien des mit der Wissenschaft in offenem Kampfe stehenden Buchstabenglaubens. Ich mufs offen gestehen, dafs mir die von $\mathrm{E}$. Krey in Hilgenfeld's Zeitschrift 1877, S. 404 ff. aufgewiesenen Zahlenspiele keineswegs den Eindruck gemacht haben, welchen sie auf Wellhausen (Bleek, S. 264 f.) und Stade (Geschichte des Volkes Israel. Berlin 1881, S. 
95 ff.) gemacht zu haben scheinen. Aehnliche Zahlenspielereien liefsen sich wol mit etwas Einbildungskraft und Scharfsinn in allen Geschichtstabellen finden, und ich erinnere auch an die zahllosen Selbsttäuschungen, denen sich der mit Zahlen spielende Hengstenberg hingegeben hat. Gewifs ist der Schlufs, dafs jede der überlieferten biblischen Königszahlen anfechtbar sei, weil sie alle dem nachexilischen chronologischen System haben dienen müssen, logisch ganz correct; als historisch einzig richtig aber gilt mir der Satz, dafs die Glaubwürdigkeit der grofsen Menge der vom Epitomator überlieferten hebräischen Königszahlen durch die Uebereinstimmung mit den gesicherten Entdeckungen der Assyriologie in befriedigender Weise gedeckt ist, sollte mein Versuch auch noch mancher Verbesserung bedürfen.

Bonn, 6. April 1883.

Die Saadjanische Uebersetzung des Hohenliedes bei Abulwalîd Merwân Ibn Ganâh nebst einigen Bemerkungen zu Merx' Ausgabe derselben.

Von Prof. Dr. Wilh. Bacher.

Die von Prof. Merx als erster Theil der Festschrift für die orientalische Section der Versammlung deutscher Philologen in Karlsruhe (Heidelberg 1882) herausgegebene arabische Uebersetzung des Hohenliedes gehört nach den in der Einleitung zu dieser Ausgabe angeführten Gründen mit grolser Wahrscheinlichkeit dem Gaon aus Fajjûm an. Abulwalîd, der hundert Jahre nach Saadja blühte, führt zwar keine Erklärung oder Uebersetzung zum Hohenliede im Namen des Gaon an, aber die nun zum ersten Male veröffentlichte Uebersetzung scheint auch von 
ihm benutzt worden zu sein, wenn auch nicht bewiesen werden kann, dafs er sie als die des Saadja kannte. Indessen beweist die Nichtnennung Saadja's in den anzuführenden Citaten aus Abulwalîd nichts gegen die Urheberschaft des ersteren. Abulwalîd, der Saadja aufser der bekannten Anrufung seiner Autorität in der Einleitung zum Kitâb-al-luma ${ }^{(1)}$ nur selten mit Namen anführt ${ }^{2}$ ), hat in seinem Wörterbuche sehr oft Saadja's Uebersetzung im Auge, wenn er a n o n y m die arabische Wiedergabe eines Bibelwortes oder Bibelsatzes anführt, gewöhnlich mit der

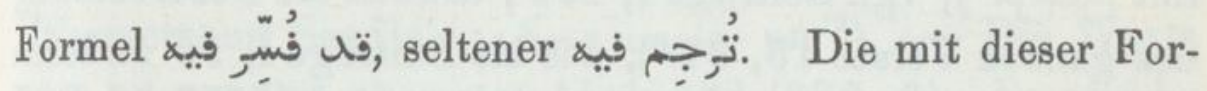
mel eingeleiteten, ziemlich zahlreichen Citate im Kitâb-aluṣal verdienen eine besondere und eingehendere Erörterung und müssen namentlich mit Saadja's Uebersetzungen biblischer Bücher, soweit diese zugänglich sind, verglichen werden. Für die Saadjanischen Uebersetzungen der Psalmen und des Hiobbuches, aus denen Ewald im I. Bande der Beiträge zur Geschichte der ältesten Auslegung und Spracherklärung des A. T. reichliche Auszüge gegeben hat, finden sich folgende Anführungen bei Abulwalîd. Im Artikel פלג sagt Abulwalîd (Wörterbuch, Col. 572, Z. 29 f.) : "Nicht erfreut mich die Erklärung (oder Uebersetzung, (تفسير (Psalm 65, 10, Jesaia 30, 25 (Antheil Gottes, Antheile) übersetzt." Das ist ein höflich eingekleideter Tadel der Ueber-

$\left.{ }^{1}\right)$ S. Munk, Notice sur Abou'l Walîd, p. 135, 140 f., 143, Riḳmâ, ed. Goldberg, p. V, VII, VIII.

$\left.{ }^{2}\right)$ Im Rikma s. p. 7,97 u. 141; im Wörterbuche Col. 111, 284, 298, 308, 349, 619, 642, 657, 726 ; im Mustalhịk (Opuscules, ed. D er e nbourg), p. 141 . 